\title{
Dielectrical properties of Hungarian acacia honeys
}

\author{
ESZTER VOZÁRY ${ }^{1^{*}}$ (D), KINGA IGNÁCZ ${ }^{2}$ and BÍBORKA GILLAY ${ }^{1}$
}

${ }^{1}$ Department of Physics and Control, Faculty of Food Science, Szent Istvan University, Budapest, Hungary

2 Törley Sparkling Wine Cellar, Budapest, Hungary

\section{CONFERENCE FULL PAPER}

Received: January 31, 2020 • Accepted: October 5, 2020

Published online: November 11, 2020

(C) 2020 The Author(s)

\section{ABSTRACT}

The statement of overheating of honey during the processing is important in quality characterization of honey products. Four Hungarian acacia honeys were heated up to $35,40,50,60$, and $80{ }^{\circ} \mathrm{C}$ and held in water bath for $0.5,4$ and $24 \mathrm{~h}$. The electrical impedance spectrum of honeys before and after heating at room temperature $\left(22^{\circ} \mathrm{C}\right)$ were measured with precision LCR meters in frequency range from $30 \mathrm{~Hz}$ up to $30 \mathrm{MHz}$ at $1 \mathrm{~V}$ voltage with $\mathrm{Ag} / \mathrm{AgCl}$ electrodes. The spectra after open-short correction were approached with a circuit model consisting of a serial connection of two distributed elements and a resistance. The model parameters were determined. One of the resistance parameters can be used for detecting the previous heating of honey after detailed investigation of the recrystallization process following the heating. The complex electrical permittivity also was determined in the frequency range from $1 \mathrm{MHz}$ up to $3 \mathrm{GHz}$.

\section{KEYWORDS}

honey, heating, electrical permittivity, electrical impedance

\section{INTRODUCTION}

Honey is a natural sweet, viscous, fluid food. It is produced from the nectars of flowers by honeybees. It contains sugars, organic acids, various amino acids, and biological active compounds: $\alpha$-tocopherol, ascorbic acids and flavonoids (Bogdanov, 2014; Turhan et al., 2008). The bee-honey is a natural food and does not allow the addition of any other substances (Diacu and

\footnotetext{
*Corresponding author. E-Mail: Vozary.Eszter@etk.szie.hu
} 
Tantaveanu, 2007). The honey from beehive is processed by several operations among others thermal treatments. At first the honey is heated up to $55^{\circ} \mathrm{C}$ for liquefaction and clarification and before packing is heated up to higher temperature for pasteurization (Subramanian et al., 2007). The heating can cause changes in the structure of proteins, can change, even can degrade the quality of honey.

As a consequence of heating the concentration of 5-hydroxymethylfurfural (HMF) can increase (Turhan et al., 2008). The permissible HMF content can be maximum $40 \mathrm{mg} / \mathrm{kg}$. Generally, the detection of HMF content can be realized by chemical investigations, which are time-consuming and chemical-intensive.

The measurement of physical properties can be realized in a short time and no chemicals are needed. During the thermal processing, the microcrystal structure of honey can change, the mobility of ions and charged groups can increase causing a decrease of electrical impedance of honey. The recrystallization of honey, the recovery to the original state presumably takes a relatively long time - some weeks, months. On the basis of this supposition the aim of this work was to answer the question: can the electrical impedance spectrum, the parameter of spectrum or the electrical permittivity be used for detecting the former heating of honey?

\section{MATERIALS AND METHODS}

Four acacia honeys - honey 1 (from Kiskunsági Nemzeti Park), honey 2 (from Örkény), honey 3 (from Baracska) and honey 4 (from Nagykáta) - were used in thermal processing. In each thermal processing a fresh (unprocessed) sample of $40 \mathrm{~g}$ was placed into a glass with cover. The glass was placed into the water bath of a thermostat - Thermo HAAKE DC10 - or into a laboratory drying oven - Venticell - for heating.

The temperature of thermostat was set to $35,40,50,60$, and $80{ }^{\circ} \mathrm{C}$ and the samples were placed into the warm water for 0.5 or $4 \mathrm{~h}$. In the experiments of $24 \mathrm{~h}$ thermal processing the glass with honey was placed into the laboratory drying oven with the given temperature for $24 \mathrm{~h}$. After heating the samples were cooled down to room temperature - to $22{ }^{\circ} \mathrm{C}$.

Electrical impedance spectrum of various honey samples was determined at room temperature before heating and after heating when the sample was cooled down for room temperature. Two Ag/ $\mathrm{AgCl}$ electrodes (World Precise Instrument) one $\mathrm{cm}$ away from each other were inserted into the honey $1 \mathrm{~cm}$ deep and the voltage between the two electrodes was $1 \mathrm{~V}$. The magnitude and the phase angle of impedance were measured in frequency range from $30 \mathrm{~Hz}$ up to $1 \mathrm{MHz}$ with a $\mathrm{HP} 4284 \mathrm{~A}$ precision LCR meter and in frequency range from $75 \mathrm{kHz}$ up to 30 $\mathrm{MHz}$ with a HP 4285A precision LCR meter. The measured spectra were open and short corrected - according to HP method - for eliminating the stray capacitance and inductance. The open and short corrected spectra were approached with an electrical circuit model consisting of serial connection of a resistance and two distributed elements (Grimnes and Martinsen, 2008):

$$
R_{0}+\frac{R_{1}}{1+\left(i \omega \tau_{1}\right)^{\psi_{1}}}+\frac{R_{2}}{1+\left(i \omega \tau_{2}\right)^{\psi_{2}}},
$$

where $R_{0}, R_{1}$ and $R_{2}$ are resistances, $i$ is the imaginary unit, $\omega=2 \pi f$ is the angular frequency, $f$ is the measuring frequency, $\tau_{1}$ and $\tau_{2}$ are relaxation times and $\psi_{1}$ and $\psi_{2}$ are exponents. The model parameters were determined with the Solver function of Excel program. 
The impedance measurement on one honey sample was repeated three times and each of the three spectra was approached with model (1) and the average and deviation of parameters were calculated.

The real $\left(\varepsilon^{\prime}\right)$ and imaginary $\left(\varepsilon^{\prime \prime}\right)$ part of complex electrical permittivity $(\varepsilon)$ also was measured with E4991A impedance analyser in frequency range from $1 \mathrm{MHz}$ up to $3 \mathrm{GHz}$ with 85070E Agilent coaxial measuring head and with the 85070D Agilent software:

$$
\varepsilon=\varepsilon^{\prime}-i \varepsilon^{\prime \prime}
$$

The measuring head before the measurement was calibrated with air and distilled water.

\section{RESULTS}

A typical measured impedance magnitude and phase angle spectrum can be seen in Fig. 1. The shape of spectra of all investigated honey samples were remarkably similar to each other, both the magnitude and the phase angle of impedance decreased with increasing frequency. All the measured impedance spectra of honey samples were approached with Eq. (1) and the model parameters were determined.

The result of curve fitting can be seen in Fig. 2 .

The model parameters in all experiments were determined for all honeys. Although all investigated honeys were acacia honey and their geographical origins are not far from each other the parameter values of untreated honeys (c) are different as it can be seen in Figs. 3-5. The tendency of parameter changes after thermal processing also shows differences for the different honeys.

The initial values of $R_{0}$ were different for the four honeys. The value of $R_{0}$ parameter increased for honey 1, practically remained unchanged for honey 2 and decreased for honey 3 and 4 as the temperature of thermal process was increased (Fig. 3 A1, B1, C1, D1).

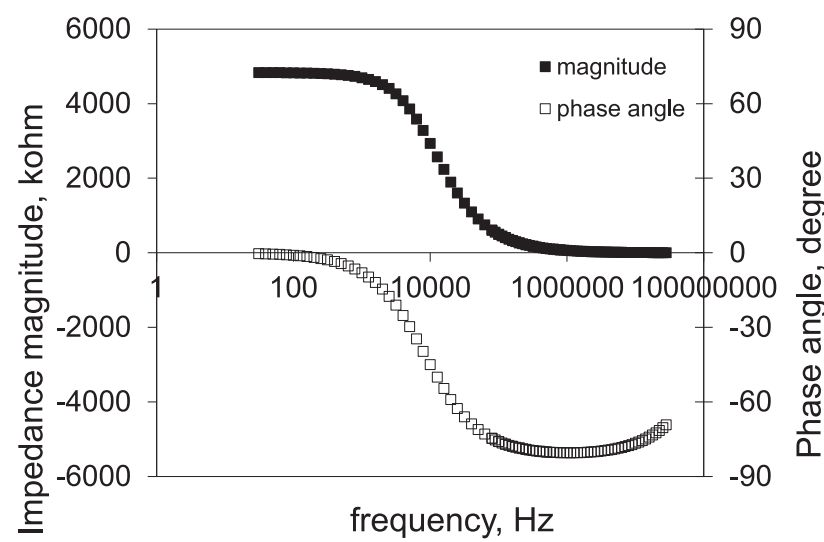

Fig. 1. The measured impedance magnitude and phase angle spectrum of untreated honey 1 (from Kiskunsági Nemzeti Park) as a function of frequency 

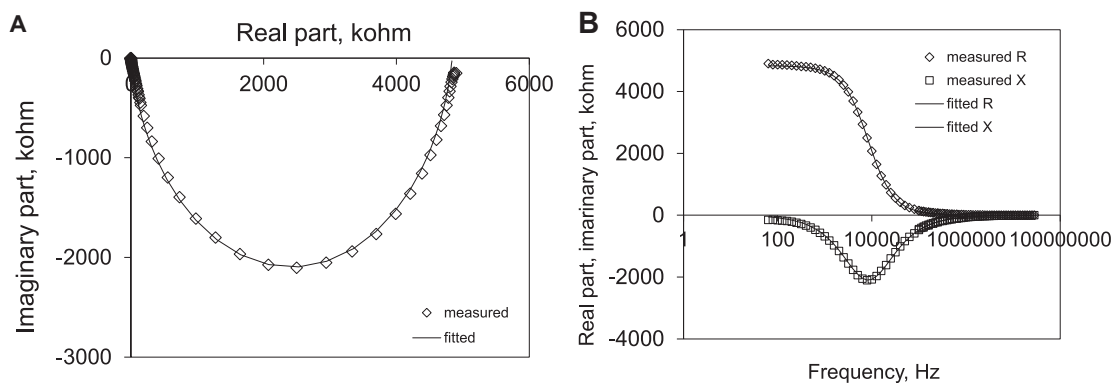

Fig. 2. Measured and fitted real and imaginary part of untreated honey 1 (from Kiskunsági Nemzeti Park) impedance on the complex plane (A) and as a function of frequency (B)

The $R_{1}$ parameter showed decreasing tendency for all processed honeys (Fig. 3 A2, B2, C2, D2) as the temperature of heating was increased, but the individual $R_{1}$ values had a large spread.

The $R_{2}$ parameter decreased for honey 1 and honey 2 (Fig. 3. A3, B3) and showed various values for honey 3 (Fig. 3 C3) and increased for honey 4 (Fig. 3 D3) with increasing processing temperature.

The initial (unheated) $\tau_{1}$ and $\tau_{2}$ values were varied from $1.5 \times 10^{-5} \mathrm{~s}$ up to $7.6 \times 10^{-5} \mathrm{~s}$ and from $5 \times 10^{-4} \mathrm{~s}$ up to $6.8 \mathrm{~s}$, respectively (Fig. 4 column "c"). The relaxation time $\tau_{1}$ had also decreasing tendency for honeys (Fig. 4 A1, B1, C1) except honey 4 (Fig. 4. D1) when the processing temperature and time were increased in the heating processes. The values of $\tau_{2}$ parameter were both lower and higher than unprocessed values for honey 1, honey 3 and honey 4 (Fig. 4. A2, C2, D2) as the processing time and temperature increased. The $\tau_{2}$ parameter showed decreasing tendency for honey 2 (Fig. 4. B2).

The initial value of exponent parameter $\psi_{1}$ was varied from 0.86 up to 0.98 (Fig. 5 A1, B1, C1, D1). After processing this parameter had changed a little. The parameter $\psi_{2}$ generally increased as the processing time and temperature increased (Fig. 5 A2, B2, C2, D2).

The real and imaginary part of electrical permittivity of acacia honeyl (Fig. 6 A and B) has a similar spectrum as can be found in scientific literature (Guo et al., 2008, 2010). The effect of heat processing on the real and the imaginary part of complex permittivity spectrum can be demonstrated with difference spectrum of "after processing minus before processing" for both parameters (Fig. $6 \mathrm{C}, \mathrm{D})$.

The other investigated honeys had similar spectra of real part and imaginary part of complex permittivity (not shown). Generally, both real and imaginary part of complex permittivity increased after thermal processing. The changes were not considerable and did not correlate with the temperature and the time of processing.

\section{DISCUSSION}

The first distributed element in Eq. (1) describes the polarization and conductance of crystals and the second distributed element describes the impedance of medium surrounding crystals. 

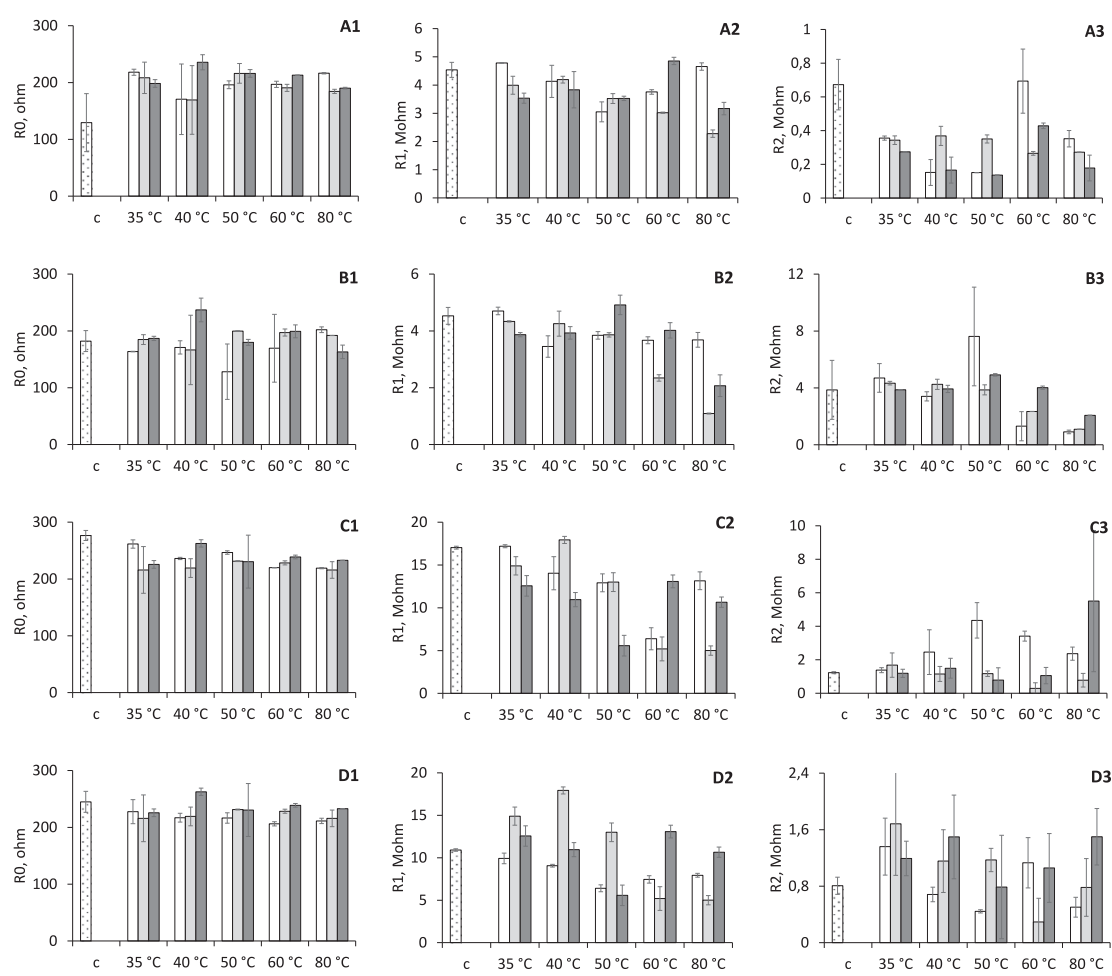

Fig. 3. The resistance parameters $-R_{0}, R_{1}, R_{2}$ - of honey1 (A), honey 2 (B), honey 3 (C) and honey 4 (D) as a function of processing temperature. The average values with standard deviation. The " $\mathrm{c}$ " denotes the parameter of unheated control sample. Heating time $0.5 \mathrm{~h}$ (white), $4 \mathrm{~h}$ (grey) and $24 \mathrm{~h}$ (black)

While the lower relaxation times and the higher exponent parameters represent the ordered crystal structure the higher relaxation times and the lower exponent parameters characterize the fluid phase. There are observations that there are crystals in honey even at high temperatures, their shape is different from the shape of crystals at low temperatures (Bakier, 2004). The parameter $R 0$ gives the resistance of honey at infinitely high frequency, the value of this parameter was slightly changed except of honey 1 (Fig. 3).

Practically all parameters were sensitive for the heating-cooling process, but whether the value of the parameter increased or decreased depended on the place of origin of the honey (Figs. 3-5).

Decreasing of $R_{1}$ parameter (Fig. 3) can be caused by the melting of some part of crystals at high temperature. The rate of decrease of $R_{1}$ was greater with longer-term and higher temperature treatments. The recrystallization process for acacia honeys is relatively slow but it begins immediately after cooling (Bhandari et al., 1999). Our measurements were executed in some cases immediately after cooling and in other cases within some days after cooling. This can cause relatively large differences in the values of the 

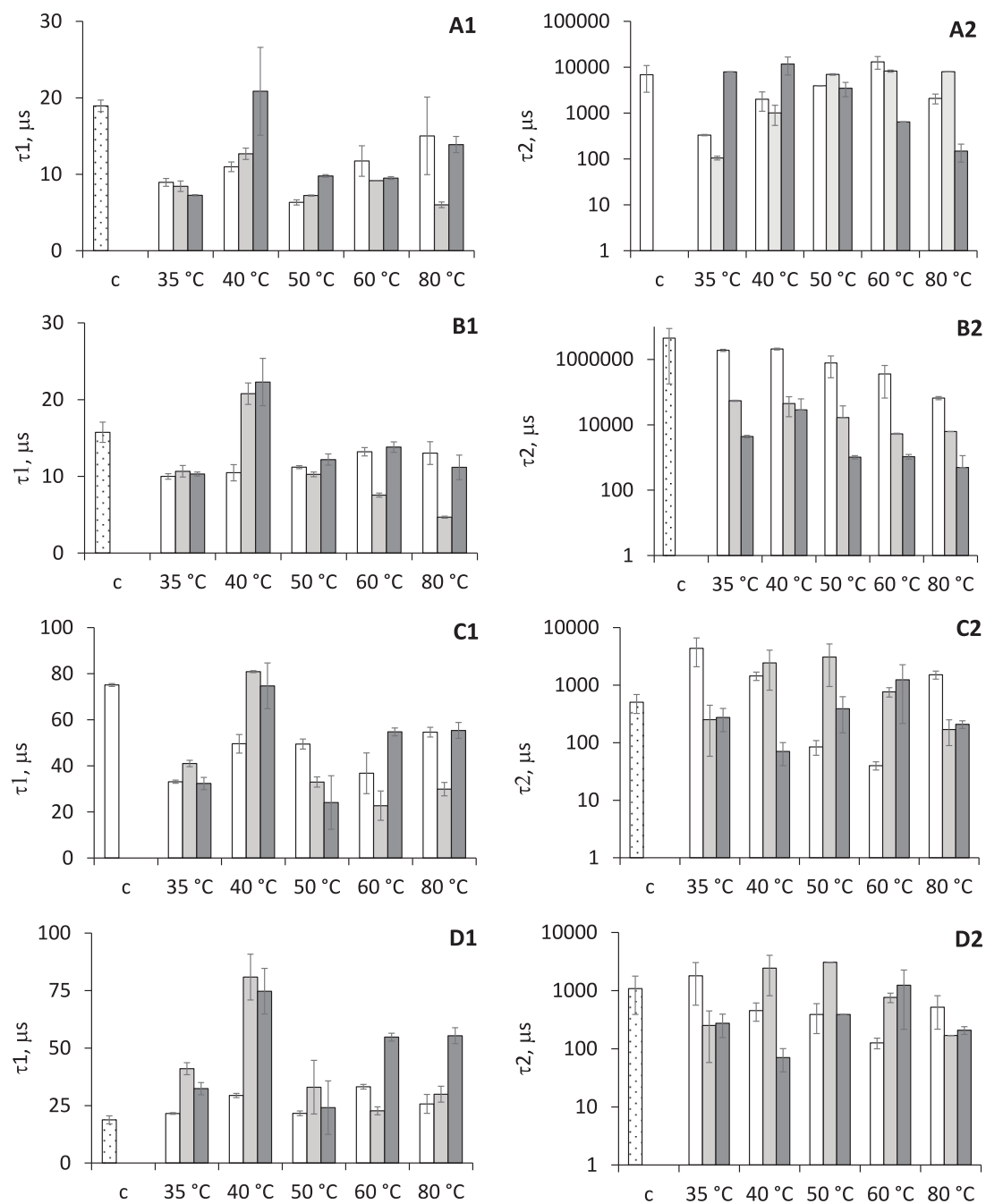

Fig. 4. The relaxation time parameters $-\tau_{1}, \tau_{2}$ - of honey 1 (A), honey 2 (B), honey 3 (C) and honey 4 (D) as a function of processing temperature. The average values with standard deviation. The "c" denotes the parameter of unheated control sample. Heating time $0.5 \mathrm{~h}$ (white), $4 \mathrm{~h}$ (grey) and $24 \mathrm{~h}$ (black)

parameters. The increase of $R_{2}$ parameter can be caused by higher concentration of melted fructose and glucose in the fluid phase, which can rise the viscosity of honey, the higher viscosity decreases the ion mobility.

The increase of viscosity can result the increasing relaxation time $\tau_{2}$ (Fig. 4). The decrease of the $\tau_{1}$ value can be the consequence of the decrease of crystal size. A small change in the 

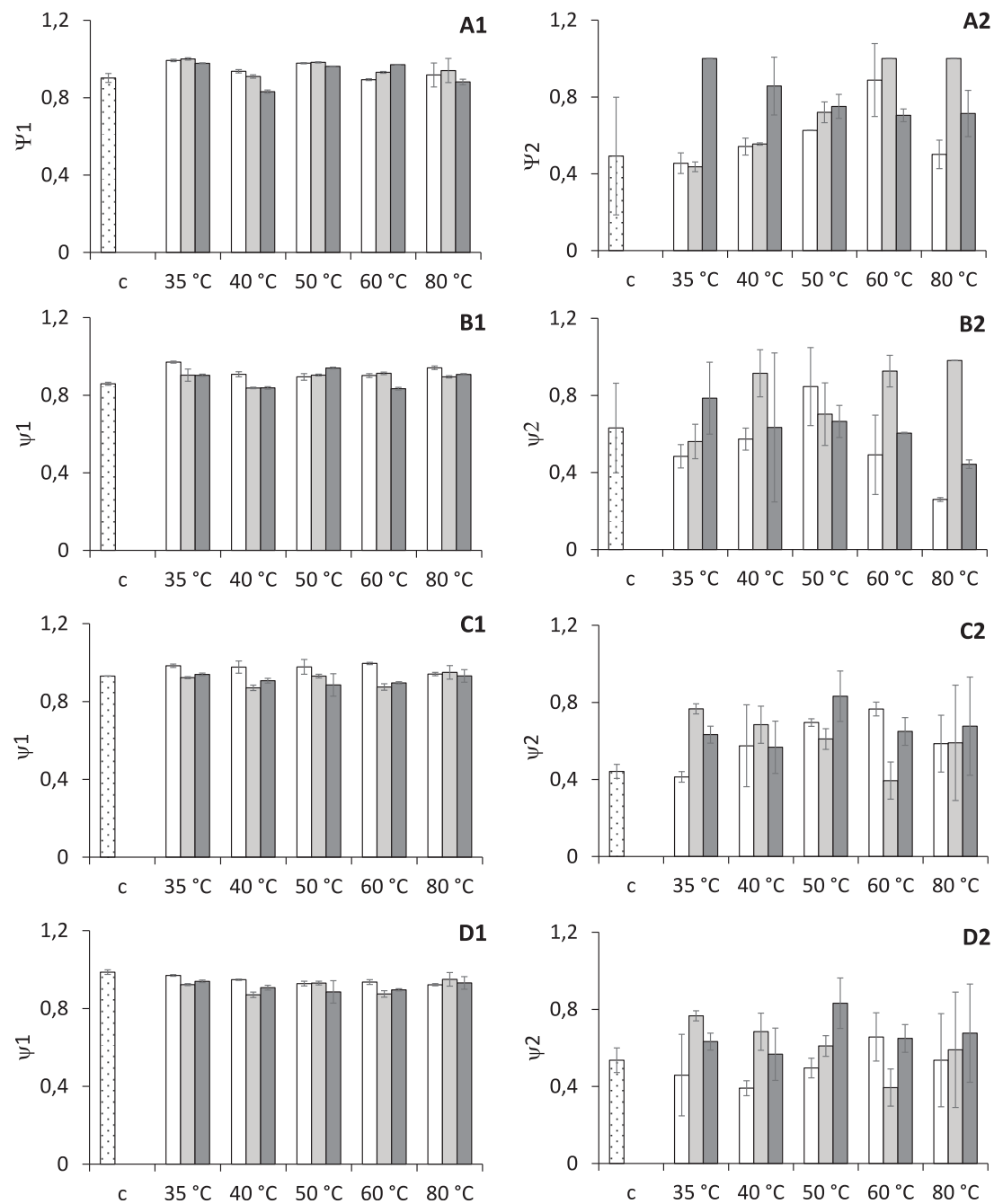

Fig. 5. The exponent parameters $-\psi_{1}, \psi_{2}$ - of honey 1 (A), honey 2 (B), honey 3 (C) and honey 4 (D) as a function of processing temperature. The average values with standard deviation. The " $\mathrm{c}$ " denotes the parameter of unheated control sample. Heating time $0.5 \mathrm{~h}$ (white), $4 \mathrm{~h}$ (grey) and $24 \mathrm{~h}$ (black)

parameter $\psi_{1}$ suggests that the nature of the electrical conduction and polarization in the crystals does not change during the partial melting of the crystals. The increase of parameter $\psi_{2}$ can be explained by the higher glucose and fructose concentration.

The increase in $\varepsilon^{\prime}$ and $\varepsilon^{\prime \prime}$ can also reflect the increased polarizability and the decreased conductivity. 

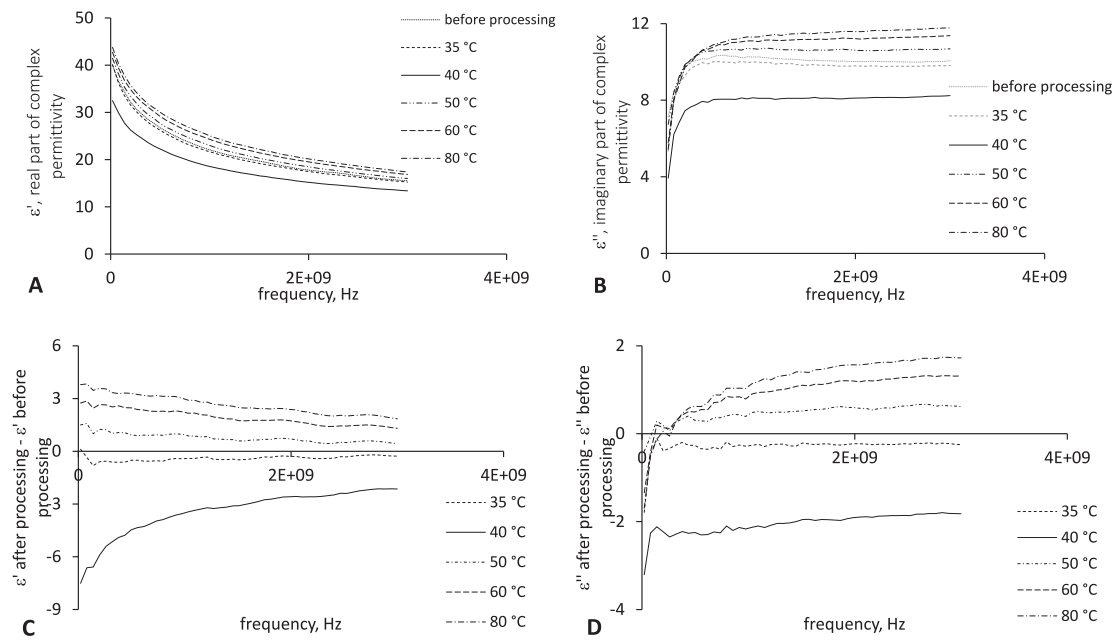

Fig. 6. The spectrum of real part (A) and imaginary part (B) of complex permittivity of honey 1 before and after thermal processing and the difference spectra (C and D) of "after processing minus before processing" at $0.5 \mathrm{~h}$ processing

\section{CONCLUSIONS}

Impedance parameter $R_{1}$ can be used to detect the previous heating of acacia honey. In order to use the impedance spectrum to detect the previous heating of honey, the process of recrystallization of honey after heating must be known in detail.

\section{REFERENCES}

Bakier, S. (2004). Description of phenomena occurring during the heating of crystallized honey. Acta Agrophysica, 3: 415-424.

Bhandari, B., D'Arcy, B., and Kelly, C. (1999). Rheology and crystallization kinetics of honey: present status. International Journal of Food Properties, 2(3): 217-226.

Bogdanov, S. (2014). Honey composition. In The honey book, Chapter 5, Bee Product Science, pp. 27-36.

Diacu, E., and Tantaveanu, E.F. (2007). Determination of moisture content and its correlation with other parameters in honey quality control. Revista de Chimie, 58(12): 1311-1312.

Grimnes, S., and Martinsen, O.G. (2008). Bioimpedance and bioelectricity basics. Elsevier, Amsterdam, New York, London.

Guo, W., Liu, Y., Zhu, X., and Wang S. (2011). Temperature-dependent dielectric properties of honey associated with dielectric heating. Journal of Food Engineering, 102: 209-216.

Guo, W., Zhu, X., Liu, Y., and Zhuang, H. (2010). Sugar and water contents of honey with dielectric property sensing. Journal of Food Engineering, 97: 275-281. 
Subramanian, R., Hebbar, H.U., and Rastogi, N.K. (2007). Processing of honey: a review. International Journal of Food Properties, 10: 127-143.

Turhan, I., Tetik, N., Karhan, M., Gurel, F., and Reyhan Tavukcuoglu, H. (2008). Quality of honeys influenced by thermal treatment. LWT-Food Science and Technology, 41(8): 1396-1399.

Open Access. This is an open-access article distributed under the terms of the Creative Commons Attribution 4.0 International License (https://creativecommons.org/licenses/by/4.0/), which permits unrestricted use, distribution, and reproduction in any medium, provided the original author and source are credited, a link to the CC License is provided, and changes - if any - are indicated. (SID_1) 